\title{
Macular Hole Closure with Minimal or No Posturing After Macular Hole Surgery
}

\author{
Muhammad Amer Awan ${ }^{1}$, Javeria Muid ${ }^{2}$, Aiman Hafeez ${ }^{3}$ \\ ${ }^{1-3}$ Department of Ophthalmology, Shifa International Hospital, Islamabad - Pakistan
}

\begin{abstract}
Purpose: To find the success rate of idiopathic and traumatic Macular hole $(\mathrm{MH})$ with minimal/ no posturing after Macular Hole (MH) surgery.

Study Design: Interventional case series.

Place and Duration of Study: Ophthalmology Clinic, Shifa International Hospital Islamabad from July 2017 to December 2018.

Material and Methods: Nineteen eyes of 19 patients with either idiopathic or traumatic $\mathrm{MH}$ were included in the study. All the patients with decreased vision due to any other cause or previous failed $\mathrm{MH}$ surgery were excluded. Their preoperative swept source OCT scans were done and $\mathrm{MH}$ was categorized according to size of $\mathrm{MH}$. Twenty seven gauge 3 port pars plana vitrectomy, inner limiting membrane peel and gas (Hexafluoroethane) tamponade was performed in all the patients. No posturing was advised for small $\mathrm{MH}$ while minimal prone positioning was advised for medium and large sized $\mathrm{MH}$.

Results: All the patients had closed $\mathrm{MH}$ at post-operative day 1 except one patient who showed decrease in size of hole after surgery. There was significant improvement in vision in all patients from mean preoperative visual acuity of 0.8 logarithm of minimum angle of resolution (Range 0.3 to 2.0) to mean post-operative visual acuity of 0.3 logarithm of minimum angle of resolution (Range 0.1 to 0.5 ). On an average 4 lines improvement in visual acuity occurred.
\end{abstract}

Conclusion: This study confirms MH closure within 24 hours on the basis of swept source OCT. Prone positioning does not appear to affect closure of small MH. For medium and large sized $\mathrm{MH}$, minimal posturing is needed.

Key Words: Macular hole, Pars plana vitrectomy, Hexafluoroethane, Optical coherence tomography.

How to Cite this Article: Awan MA, Muid J, Hafeez A. Macular Hole Closure with Minimal or No Posturing After Macular Hole Surgery, Pak J Ophthalmol. 2020; 36 (1): 8-12.

DOI: https://doi.org/10.36351/pjo.v36i1.940

\section{INTRODUCTION}

Macular hole (MH) is a full thickness break in the neurosensory retina that involves the center of macula ${ }^{1}$. MHs have an incidence rate of 7.8/1000,000 and they more commonly affect elderly females ${ }^{2}$. Most common etiological factors are idiopathic and trauma.

Correspondence to: Muhammad Amer Awan

Consultant Ophthalmologist

Shifa International Hospital, Islamabad, Pakistan

Email:dramer_awan@yahoo.co.uk
Various theories have been proposed in the pathogenesis of idiopathic $\mathrm{MH}$ but over the past decade, it has been accepted worldwide that vitreomacular traction at the fovea is the leading cause ${ }^{3}$. Therefore, treatment is usually surgical that is 3-ports pars plana vitrectomy with internal limiting membrane peel and gas tamponade. It has a success rate of over 90 percent $^{1}$. Five years follow up of the patients has shown that 58 percent of the patients achieve visual acuity of 20/40 or better and 77 percent of patients gain 3 or more lines of Snellen visual acuity chart ${ }^{4}$. Prone positioning is recommended by vitreoretinal surgeons after MH sur- 
gery and is considered as an important factor in closure of $\mathrm{MH}^{5}$. Yorston et al showed comparable results in posturing and non-posturing groups in a randomized controlled trial and suggested that face down posturing is not required for small and medium sized $\mathrm{MH}^{6}$. Prone positioning can be very inconvenient for the patients because of aging, breathing problems, obesity and arthritis ${ }^{4,5}$. Furthermore, detailed examination of the retina in the early postoperative period after $\mathrm{MH}$ surgery is very hard because of intraocular gas tamponade ${ }^{7}$.

Optical coherence tomography (OCT) has emerged from an experimental instrument to an important non-invasive imaging tool providing us the three dimensional cross sectional view of the retina and giving us the minute details of ellipsoid zone and myoid zone $^{8}$. Intact ellipsoid zone is a prognostic factor for post-operative visual improvement ${ }^{9}$. Tomographic images of swept source OCT in early post-operative period (within 24 hours) enables us to see the $\mathrm{MH}$ status in gas-filled eyes, thus allowing early discontinuation of, or no necessity for, prone positioning upon confirmation of MH surgery to see the layers of retina and to debate on whether prone positioning is mandatory or $\operatorname{not}^{10}$.

The purpose of our study was to evaluate MH closure on OCT within 24 hours of surgery, at 1 week and at 8 weeks and to identify the success rate of macular hole surgery with minimal/ no posturing in idiopathic and traumatic $\mathrm{MH}$.

\section{MATERIAL AND METHODS}

The institutional review board approval for the study was taken and it was conducted in ophthalmology clinic, Al-Shifa International Hospital Islamabad. Consecutive sampling technique was used that included 19 eyes of 19 patients from July 2017 to December 2018.

Patients with idiopathic $\mathrm{MH}$ and traumatic $\mathrm{MH}$ with or without cataract were included in the study. All the patients with decreased vision due to any other cause or previous failed MH surgery were excluded. DRI OCT Triton, Swept Source OCT (SS-OCT), Topcon was used for this study. After taking informed written consent, patients had their preoperative OCT scans done and size of the base of MH was measured. Patients were categorized into three groups according to the size of MH.

Closure of $\mathrm{MH}$ on OCT was defined as:
- Type 1 closure: Closed MH without foveal neurosensory retinal defect.

- Type 2 closure: Closed MH with foveal neurosensory retinal defect, which shows decrease in size of base of defect as measured on OCT.

Primary outcome measure was closure of $\mathrm{MH}$ as proved by OCT. Secondary outcome measure was visual improvement as measured by logarithm of minimum angle of resolution scale at 2 months postoperatively.

Twenty seven gauge 3 ports pars plana vitrectomy, internal limiting membrane peel and gas tamponade with C2F6 (Hexafluoroethane) was performed in all the patients. In patients with cataract, combined phacoemulsification and vitrectomy was performed. Patients were advised prone positioning if required immediately after the surgery, as shown in table 1. After which all the patients were advised not to lie on their back for 2 weeks. Follow up visits were done at 1 day, 1 week, 1 month and 2 months post operatively. OCT scans were done at $1^{\text {st }}$ post-operative day and then were repeated at $1^{\text {st }}$ and $8^{\text {th }}$ post-operative weeks to confirm the closure of macular hole.

\section{RESULTS}

There were 19 patients with female to male ratio of 12:7. Mean age was 60 years that ranged between 1977 years. Seventeen patients had idiopathic and 2 had traumatic MH. Categories of the patients were as follows:

- Category A (MH less than $400 \mu \mathrm{m}): 6$ patients.

- Category B (MH 400-700 $\mu \mathrm{m}): 4$ patients.

- Category C: MH greater than $700 \mu \mathrm{m}): 9$ patients.

Table 1: Prone positioning in patients according to the size of macular hole.

\begin{tabular}{|c|c|c|c|}
\hline $\begin{array}{l}\text { Serial } \\
\text { No }\end{array}$ & Macular Hole Size & Posture & Duration \\
\hline 1. & $\mathrm{MH}<400 \mu \mathrm{m}$ & $\begin{array}{l}\text { No posture } \\
\text { advised }\end{array}$ & Nil \\
\hline 2. & MH $400 \mu \mathrm{m}-700 \mu \mathrm{m}$ & Face down & 4-6 hours \\
\hline 3. & $\mathrm{MH}>700 \mu \mathrm{m}$ & Face down & 12 hours \\
\hline
\end{tabular}

Sixteen patients were phakic and 3 were pseudophakic. In 5 patients vitrectomy was performed and remaining 14 patients had phacoemulsification plus pars plana vitrectomy was done. There was no per- 


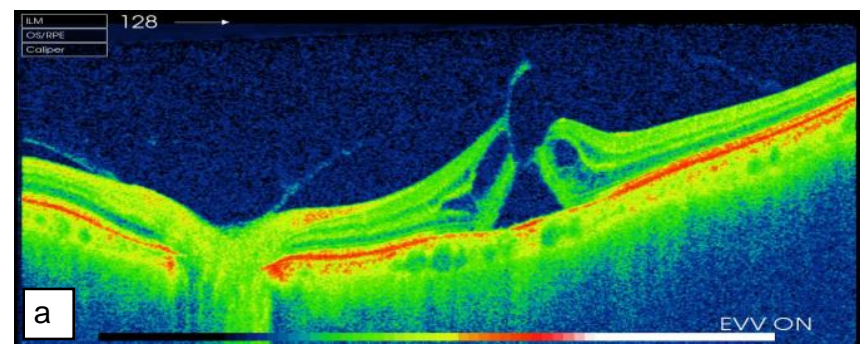

Fig. 1a: Swept source optical coherence tomography (OCT) of the left eye that shows small stage 3 full thickness macular hole $(\mathrm{MH})$.

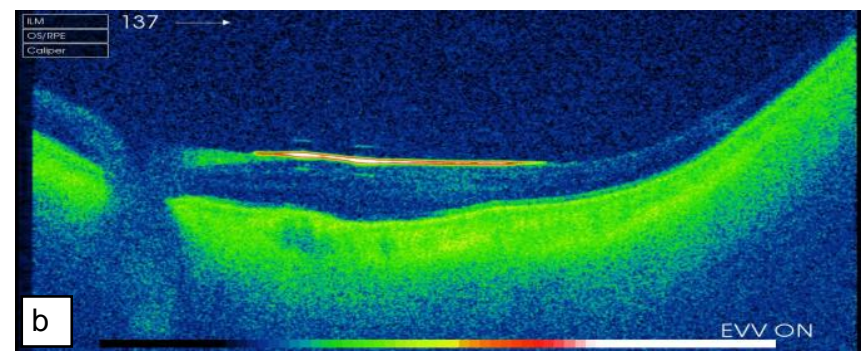

Fig. 1b: Swept source OCT on first post-operative day that depicts closure of $\mathrm{MH}$ in the left eye with very good view in spite of gas bubble.

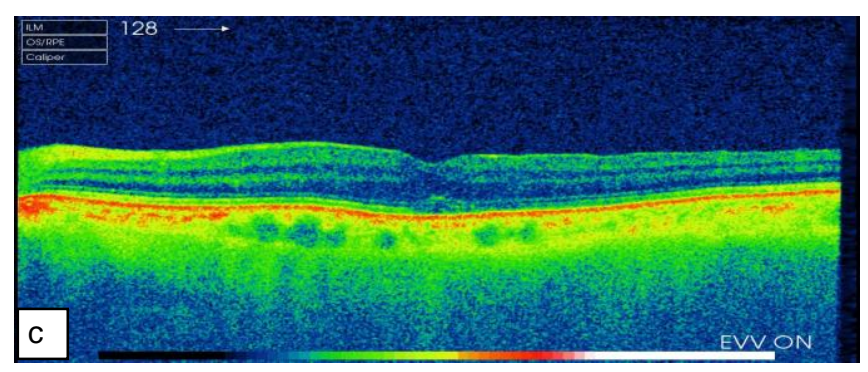

Fig. 1c: Swept source OCT of the left eye after 8 weeks that confirms closure of $\mathrm{MH}$. operative complication. At first post-operative day, all the patients who had closed MH on OCT, were advised to stop the prone positioning. All the idiopathic MH were closed on first postoperative day and one traumatic MH was not closed, as seen on OCT. Scan was further repeated at 1 and 8 weeks, as shown in figure $1 \mathrm{a}, 1 \mathrm{~b}$ and $1 \mathrm{c}$. All the patients had closed $\mathrm{MH}$ (Table 2) except one patient who showed type 2 closure of $\mathrm{MH}$ with decrease in size of macular hole. Later this patient had vitrectomy, ILM patch graft and C3F8 (Octafluoropropane) gas tamponade that closed $\mathrm{MH}$ with improvement in vision from 2 to 0.5 logarithm of minimum angle of resolution. This patient was advised to do prone positioning 6 hours daily for 1 week. There was significant improvement in vision in all patients from mean preoperative visual acuity of 0.8 logarithm of minimum angle of resolution (Range 0.3 to 2.0 ) to mean post-operative visual acuity of 0.3 logarithm of minimum angle of resolution (Range 0.1 to 0.5$)$.

The limitation of this study is the small sample size. Further research can be planned by comparing the results of prone posturing versus non-prone posturing and large sample size.

Table 2: Postoperative closure of macular hole at post op day 1, week 1 and week 8.

\begin{tabular}{|c|c|c|c|c|c|c|c|}
\hline $\begin{array}{l}\text { S. } \\
\text { No }\end{array}$ & Macular Hole Size & Category & Type of MH & $\begin{array}{l}\text { No. of } \\
\text { Patients }\end{array}$ & $\begin{array}{c}\text { Macular } \\
\text { Hole on } \\
\text { POD } 1 \\
\end{array}$ & $\begin{array}{c}\text { Macular } \\
\text { Hole on } \\
\text { POD } 8 \\
\end{array}$ & $\begin{array}{c}\text { Macular Hole after } 8 \\
\text { Weeks }\end{array}$ \\
\hline 1. & $\mathrm{MH}<400 \mu \mathrm{m}$ & A & Idiopathic & 5 & closed & Closed & Closed \\
\hline 2. & MH $400 \mu \mathrm{m}-700 \mu \mathrm{m}$ & $\mathrm{B}$ & Idiopathic and traumatic & 5 & closed & Closed & Closed \\
\hline 3. & $\mathrm{MH}>700 \mu \mathrm{m}$ & $\mathrm{C}$ & Idiopathic & 8 & closed & Closed & Closed \\
\hline 4. & $\mathrm{MH}>700 \mu \mathrm{m}$ & $\mathrm{C}$ & Traumatic & 1 & Flat hole & Flat hole & $\begin{array}{l}\text { Flat hole (Type II closure) } \\
883 \mu \mathrm{m} \text { to } 312 \mu \mathrm{m} \\
\text { Closed after further surgery }\end{array}$ \\
\hline
\end{tabular}

MH: Macular Hole; POD: Postoperative Day

\section{DISCUSSION}

In this study, the primary $\mathrm{MH}$ closure rate is 95 percent, which is comparable to international results. However, the final closure rate is 100 percent and the study also shows significant improvement in visual acuity which is an average of 4 lines on Snellen's chart. It is better than the previously reported international studies. SS-OCT also confirmed the closure of $\mathrm{MH}$ on the first post-operative day in 18 eyes that led to no further prone positioning. These facts clearly 
show that prone positioning is probably not an important factor in determining the favorable outcome. It explains that prone positioning is not required for $\mathrm{MH}$ less than $400 \mu \mathrm{m}$ and minimal positioning is required for medium and large MH. Previous studies have shown mixed results. Some of them reported better outcome with prone positioning while others revealed no difference in overall results in prone positioning group versus non-supine positioning group. One possible explanation for this can be that the patients might not be following the prone positioning instructions properly as it is difficult for them to do it because of obesity, asthma or other health issues.

In a recent study, $\mathrm{MH}$ closure rates were $95.5 \%$ and $96.4 \%$ in the prone posturing group as compared to non-supine posturing group, respectively. Median visual acuity at 6 months post operatively was 69 early treatment diabetic retinopathy study (ETDRS) letters in both the groups $(p=0.64)^{11}$. However, a meta analysis showed that prone posturing after $\mathrm{MH}$ surgery does improve the overall $\mathrm{MH}$ closure rate. Combining cataract surgery with vitrectomy does not affect the closure rate ${ }^{2}$. Another survey of American Society of Retina Specialists has revealed that 95 percent of retinal surgeons still advise prone positioning in the treatment of $\mathrm{MHs}^{7,8}$.

The prognosis of idiopathic $\mathrm{MH}$ is predictable by seeing the details of layers of central retina on OCT, size of macular hole and its duration. Closure of these types of MH as well as visual upgradation can be foreseen preoperatively but for traumatic macular holes the prognosis is variable. Some have shown significant improvement and spontaneous closure while others may show incomplete closure with little betterment when surgery is performed after 3 months of trauma ${ }^{12}$. It certainly depends upon the duration and structures of inner retina.

Lindtjørn et al strongly proposed that non-supine positioning provided excellent closure of idiopathic macular holes. He also suggested that complete gasfoveal contact without minor interruptions was not necessary for macular hole closure ${ }^{13}$. Contrary to that another researcher suggested that degree of gas fill had considerable effect on the results of hole closure ${ }^{14}$.

Recently, SS-OCT enables tomographic images of $\mathrm{MH}$ in gas-filled eyes immediately postoperatively, thus permitting early discontinuation of face-down positioning upon confirmation of MH closure ${ }^{15}$.

However, a meta-analysis of 181 records with 726 cases had shown that face down posturing was more effective in macular hole closure when compared with the non-posturing cases, especially in patients with MH larger than $400 \mu \mathrm{m}^{2}$.

There are multiple factors, which affect closure of macular hole after surgery. One is the gas tamponade for which some authors have suggested additional gas injection in case of non-closure at first attempt ${ }^{16}$. In addition to that Emi Morimoto showed that $50 \%$ of the patients are non-compliant regarding face down posturing $^{17}$. Some studies have indicated the non-supine posturing was equally good for macular hole closure ${ }^{18}$. To increase patient compliance issues, Nadal $\mathrm{J}$ presented the idea of isolating macula with a large long lasting gas, which can help macular closure without posturing ${ }^{19}$. To increase the patient compliance, another researcher suggested a regimen of ILM peel, 20\% C2F6 with a one night prone posturing ${ }^{20}$.

With advancement in OCT technology, SS-OCT is a wonderful tool to assess $\mathrm{MH}$ closure or macular attachment within few hours after surgery in a gas/oil filled eyes. This study clearly shows the benefit of swept source OCT in assessing earlier closure of $\mathrm{MH}$ after surgery and justifying no need for extensive prone positioning. However, SS-OCT is relatively difficult to perform in gas-filled eyes and experienced personnel is required for this purpose to adjust the focusing.

\section{CONCLUSION}

Prone positioning does not affect the rate of closure of MH with size less than $400 \mu \mathrm{m}$. For medium and large sized MH, posturing can be stopped within 24 hours of surgery after confirming hole closure on swept source OCT.

\section{Ethical Approval}

The study was approved by the Institutional review board/Ethical review board.

\section{Conflict of Interest}

Authors declared no conflict of interest

\section{Authors' Designation and Contribution}

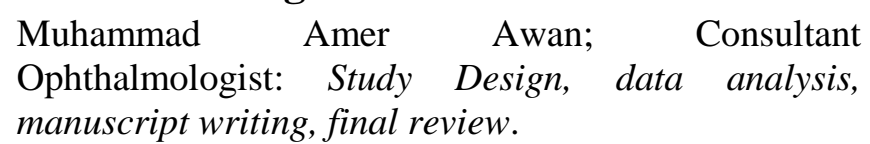


Javeria Muid; Medical Officer: Data analysis, manuscript writing, final review.

Aiman Hafeez; Optometrist: Data Collection, final review.

\section{REFERENCES}

1. Kang SW, Ahn K, Ham DI. Types of macular hole closure and their clinical implications. Br J Ophthalmol. 2003; 87 (8): 1015-9.

2. Xia S, Zhao XY, Wang EQ, Chen YX. Comparison of face-down posturing with nonsupine posturing after macular hole surgery: a meta-analysis. BMC Ophthalmol. 2019; 19 (1): 34.

3. Hu Z, Xie $\mathbf{P}$, Ding $\mathbf{Y}$, Zheng $\mathbf{X}$, Yuan D, Liu $\mathbf{Q}$. Face-down or no face-down posturing following macular hole surgery: a meta-analysis. Acta Ophthalmol. 2016; 94 (4): 326-33.

4. Patel S, Sternberg Jr P. Face-Down Positioning Following Macular Hole Surgery: Pros and Cons. J Vitreoretin Dis. 2017; 1: 331-3.

Doi: https://doi.org/10.1177/2474126417715368

5. Shimada Y, Seno Y, Mizuguchi T, Tanikawa A, Horiguchiet M. Patient adherence to the face-down positioning after macular hole surgery. Clin Ophthalmol. 2017; 11: 1099.

6. Yorston D, Siddiqui MA, Awan MA, Walker S, Bunce C, Bainbridge JW. Pilot randomised controlled trial of face-down posturing following phacovitrectomy for macular hole. Eye, 2012; 26 (2): 267.

7. Jumper JM, Gallemore RP, Toth CA. Features of macular hole closure in the early postoperative period using optical coherence tomography. Retina 2000; 20 (3): 232-7.

8. Ehlers JP, Goshe J, Dupps WJ, Kaiser PK, Singh RP, Gans R, Eisengart J, et al. Determination of feasibility and utility of microscope-integrated optical coherence tomography during ophthalmic surgery: the DISCOVER Study RESCAN Results. JAMA Ophthalmol. 2015; 133 (10): 1124-32.

9. Kothari N, Tran KD, Read SP, Berrocal AM. Optical Coherence Tomography Imaging After Surgical Closure of Pediatric Traumatic Macular Holes Optical
Coherence Tomography Imaging After Surgical Closure of Pediatric Traumatic Macular Holes. J Vitreoretin Dis. 2018; 2: 104-6.

Doi: https://doi.org/10.1177/2474126417749320

10. Ehlers JP, Uchida A, Srivastava SK, Hu M. Predictive Model for Macular Hole Closure Speed: Insights from Intraoperative Optical Coherence Tomography. Transl Vis Sci Technol. 2019; 8 (1): 18.

11. Alberti M, la Cour M. Face-down positioning versus non-supine positioning in macular hole surgery. $\mathrm{Br} J$ Ophthalmol. 2015; 99 (2): 236-9.

12. Miller JB, Yonekawa Y, Eliott D, Kim IK, Kim LA, Loewenstein JI, et al. Long-term follow-up and outcomes in traumatic macular holes. Am J Ophthalmol. 2015; 160: 1255-8.e1.

13. Lindtjorn B, Krohn J, Austeng D, Fossen K, Varhaug P, Basit S, et al. Nonsupine Positioning after Macular Hole Surgery: A Prospective Multicenter Study. Ophthalmol Retina. 2019; 3 (5): 388-92.

14. Alberti M, La Cour M. Nonsupine positioning in macular hole surgery. Retina 2016; 36 (11): 2072-9.

15. Kikushima W, Imai A, Toriyama Y, Hirano T, Murata $\mathbf{T}$, Ishibashi $\mathbf{T}$. Dynamics of macular hole closure in gas-filled eyes within $24 \mathrm{~h}$ of surgery observed with swept source optical coherence tomography. Ophthalmic Res. 2015; 53 (1): 48-54.

16. Iwase T, Sugiyama K. Additional gas injection after failed macular hole surgery with internal limiting membrane peeling. Clin Exp Ophthalmol. 2007; 35 (3): 214-9.

17. Morimoto E, Shimada Y, Sugimoto M, Mizuguchi T, Tanikawa A, Horiguchi M. Adherence to face-down and non-supine positioning after macular hole surgery. BMC Ophthalmol. 2018; 18 (1): 322.

18. Forsaa VA, Raeder S, Hashemi LT, Krohn J. Shortterm postoperative non-supine positioning versus strict face-down positioning in macular hole surgery. Acta Ophthalmol. 2013; 91 (6): 547-51.

19. Nadal J, Delas B, Pinero A. Vitrectomy without facedown posturing for idiopathic macular holes. Retina. 2012; 32 (5): 918-21.

20. Malik A, Dooley I, Mahmood U. Single night postoperative prone posturing in idiopathic macular hole surgery. Eur J Ophthalmol. 2012; 22 (3): 456-60. 